\title{
Percepção da Efetividade dos Métodos de Ensino Utilizados em um Curso de Medicina do Nordeste do Brasil
}

\author{
Perception of Effectiveness of the Teaching \\ Methods Used in a Medical Course in Northeast \\ Brazil
}

\section{PALAVRAS-CHAVE}

- Educação Médica.

- Ensino.

- Capacitação Profissional.
Poliana Oliveira da Cruz ${ }^{I}$ Thaís Bandeira de Carvalho ${ }^{I}$

Luca Di Pace Pinheiro

Patrícia Estela Giovannini ${ }^{I}$

Ellany Gurgel Cosme do Nascimento ${ }^{I}$

Thales Allyrio Araújo de Medeiros Fernandes ${ }^{I}$

\section{RESUMO}

A educação médica se encontra em um processo de transformação, principalmente no que se refere à diversificação dos métodos de ensino utilizados. No entanto, a utilização e correta implementação destas novas metodologias ainda constituem verdadeiros desafios. Nessa perspectiva, o presente estudo objetivou avaliar a percepção e opinião dos professores e alunos quanto à efetividade dos métodos de ensino utilizados no curso de Medicina da Universidade do Estado do Rio Grande do Norte (UERN). Para isso, foi realizado um estudo descritivo exploratório, de caráter transversal, cuja coleta de dados ocorreu de julho de 2015 a junho de 2016. Foram entrevistados 140 alunos (82,5\% dos discentes do curso) e 80 professores (55\% do corpo docente) de ambos os sexos e provenientes de todos os períodos e componentes curriculares. Após aceitação do convite para participar da pesquisa, foi realizada uma entrevista com perguntas objetivas e subjetivas, destinada à obtenção de dados sociodemográficos e opiniões e percepções dos indivíduos quanto aos métodos de ensino-aprendizagem utilizados pelo corpo docente. A análise dos resultados ocorreu por estatística descritiva e teste do $\chi 2$ de Pearson ou Exato de Fisher, utilizando-se os softwares Pepi e Past, considerando-se como significantes valores de $p \leq 0,05$. As opiniões e sugestões dos entrevistados foram representadas por meio de nuvem de palavras, utilizando-se o software WordArt. As metodologias tradicionais constituíram as técnicas mais utilizadas (76,0\% dos professores), além de serem consideradas mais eficazes do que as metodologias contemporâneas, tanto pelos professores $(78,0 \%)$ quanto pelos alunos $(92,0 \%)$. No entanto, grande parte desses (75,0\% dos docentes e 73,6\% dos discentes) gostaria de utilizar e vivenciar outras metodologias didático-pedagógicas como forma de melhorar o aprendizado. Observou-se ainda que o tempo de experiência docente e a realização de pós-graduação stricto sensu estiveram significantemente associados à utilização de métodos de ensino-aprendizagem mais contemporâneos. Diante disso, conclui-se que a falta de experiência e de vivência com outras metodologias de ensino, somada à carga horária extenuante do curso, pode dificultar a implementação e aceitação dessas metodologias mais contemporâneas. Desta forma, nota-se a premente necessidade de incentivar a capacitação didático-pedagógica do corpo docente, assim como a implementação do projeto pedagógico do curso a fim de promover o desenvolvimento de metodologias centradas no aluno, conforme recomendações atuais para o ensino dos cursos da área da saúde, principalmente o de Medicina. 


\section{KEY-WORDS}

- Medical Education.

- Teaching.

- Professional Training.

Recebido em: 26/10/18

Aceito em: 29/10/18

\begin{abstract}
Medical education is in a process of transformation, particularly in terms of the diversification of the teaching methods used. However, the use and correct implementation of these new methods still present real challenges. In this context, this study aims to evaluate the perceptions and opinions of teachers and students regarding the effectiveness of the teaching methods used on the Universidade do Estado do Rio Grande do Norte (UERN) medical course. For this purpose, an exploratory descriptive study was carried out, with a transversal character. Data collection was carried out from July 2015 to June 2016. A total of 140 students (82.5\% of the medical course students) and 80 teachers (55\% of the faculty) were interviewed. The subjects were of both sexes, and from all periods and curricular components of the course. After reading and signing a consent form, the participants were interviewed, with objective and subjective questions that sought to obtain their sociodemographic data, and their opinions and perceptions of the teaching-learning methods used by the teaching staff. The results were submitted to descriptive statistics and Pearson's $\chi 2$ test or Fisher's Exact, using the PEPI and PAST software, and considering values of $p \leq 0.05$ as significant. The interviewees' opinions and suggestions were represented in the form of a word cloud, using WordArt software. Traditional methods were the most frequently used techniques (76.0\% of teachers), and were considered more effective than contemporary methods by both the teachers $(78.0 \%)$ and the students (92.0\%). However, a high proportion of the interviewees (75.0\% of the teachers and $73.6 \%$ of the students) said that they would like to use and experience other didactic-pedagogical methods as a way to improve learning. It was also observed that length of teaching experience, and whether or not the teacher held a postgraduation qualification, were significantly associated with the use of more contemporary teaching-learning methods. It is therefore concluded that lack of teaching experience and experience with other teaching methodologies, together with the strenuous workload of the course, can pose hindrances to the implementation and acceptance of more contemporary methodologies. We note the urgent need to promote didactic-pedagogical training for the teaching staff, as well as the implementation of the Pedagogical Project of the Course, in order to promote the development of student-centered methodologies, in line with current teaching recommendations for courses in the area of health, especially Medicine.
\end{abstract}

\section{INTRODUÇÃO}

O profissional de saúde deve ser capaz de criar, planejar, implementar e avaliar políticas e ações que visem ao bem-estar geral de determinada comunidade. Essas habilidades devem ser construídas e aprimoradas ao longo da graduação, de forma que o sujeito egresso já consiga dominar suas competências para além do domínio técnico-científico e que o conteúdo aprendido seja transmitido e incorporado pelos cidadãos, numa constante situação de empoderamento da população ${ }^{1}$.

No modelo tradicional ainda predominante, a formação tem por base uma pedagogia que não estimula adequadamente a autonomia, a capacidade de análise, julgamento e avaliação, bem como raciocínio crítico, investigativo e criativo². Nesse diapasão, os métodos tradicionais de ensino são caracterizados pela transmissão verticalizada de conhecimentos e pela ênfase na memorização em detrimento da reflexão crítica, baseando-se, essencialmente, em aulas expositivas, em que o professor é o detentor de conhecimentos e grande protagonista, cabendo aos estudantes repetir fidedignamente os conteúdos memorizados nas provas classificatórias ${ }^{3}$. Esse panorama, no qual o aluno atua quase sempre de forma passiva, dificulta a sua participação no processo de busca e sedimentação do conhecimento, elemento essencial para o desenvolvimento de mentes críticas e inovadoras ${ }^{4}$.

Assim, é preciso acreditar e vivenciar um modelo educacional que não busque apenas a reorientação no que se refere à aquisição de conhecimentos e ao desenvolvimento das habilidades técnicas, mas que possibilite o exercício de habilidades sociais, de ações críticas e éticas que impulsione os alunos a rever paradigmas, contextualizando-os com a sociedade con- 
temporânea ${ }^{5}$. Além do mais, a informação é onipresente, e a troca de informações simples tem um valor relativamente baixo; em seu lugar, a sabedoria partilhada e a oportunidade de se envolver na resolução de problemas na prática são formas relevantes e tornam-se fundamentais ${ }^{6}$.

Neste contexto, o ensino nos cursos da área da saúde se encontra em um processo de transformação, inclusive no que se refere à diversificação dos métodos de ensino-aprendizagem, que utilizam diferentes abordagens, como mapas conceituais, estudos dirigidos, atividades lúdicas, aprendizado baseado em problemas (ABP), aprendizado baseado em equipes $(\mathrm{ABE})$, sala de aula invertida, entre outras. Todas essas técnicas didático-pedagógicas têm por objetivo permitir uma construção mútua de conhecimentos com base na vivência de situações reais, contrapondo-se à mera memorização e transferência verticalizada de conhecimentos. Além disso, baseiam-se em estratégias de ensino fundamentadas na concepção pedagógica crítico-reflexiva, que permite olhar e intervir sobre a realidade, favorecendo a construção coletiva do conhecimento e seus diferentes saberes ${ }^{7}$.

Entretanto, estudos mostram que a utilização de metodologia ativa também pode apresentar potenciais desvantagens, como o fato de consumir enorme tempo docente de preparo, aplicação e avaliação da atividade, requerer o trabalho com pequenos grupos para que seja efetiva, além da necessidade de selecionar o "conteúdo essencial" que será trabalhado 8 .

Até o momento, poucos trabalhos têm se dedicado a avaliar e comparar a aceitação e percepção da efetividade dos diferentes métodos de ensino-aprendizagem por parte do corpo discente e docente dos cursos da área de saúde. Diante disso, o presente estudo objetivou avaliar a percepção e opinião do corpo docente e discente quanto à efetividade dos métodos de ensino utilizados pelo corpo docente do curso de Medicina da Universidade do Estado do Rio Grande do Norte (UERN).

\section{MATERIAIS E MÉTODOS}

O trabalho consistiu em um estudo descritivo exploratório, de caráter transversal, cuja coleta de dados ocorreu no período de julho de 2015 a junho de 2016. A pesquisa tinha como público-alvo o universo dos discentes (254) e docentes (97) vinculados ao curso de graduação em Medicina da Universidade do Estado do Rio Grande do Norte (UERN), localizada no município de Mossoró (RN). Na ocasião, foram entrevistados 140 alunos (55,1\% do corpo discente) e 80 professores (82,5\% do corpo docente) de ambos os sexos e provenientes de todos os períodos / componentes curriculares do curso de Medicina da UERN.

Os critérios para inclusão dos discentes no estudo foram: ser maior de idade, estar regularmente matriculado no curso de
Medicina da UERN, frequentar normalmente as aulas ou participar das atividades de estágio curricular obrigatório, estar presente nas dependências da Faculdade de Ciências da Saúde (Facs) da UERN durante as visitas destinadas à coleta de dados e aceitar participar do estudo. Além desses, foram incluídos os professores, efetivos ou provisórios, vinculados à Facs-UERN, em efetivo exercício da atividade docente, que ministravam aulas em qualquer período do curso de Medicina, que aceitaram participar do estudo e estavam presentes nas dependências da unidade acadêmica durante as visitas destinadas à coleta de dados. Foram excluídos os alunos ou docentes que formalmente manifestaram o desejo de ter seus dados retirados do estudo.

Todos os indivíduos foram previamente esclarecidos quanto aos objetivos do trabalho e procedimentos a serem adotados e, após aceitarem o convite para participar do estudo, assinaram o Termo de Consentimento Livre e Esclarecido (TCLE). Em seguida, foram aplicados questionários padronizados (um para os discentes e outro para os docentes), constituídos de perguntas abertas e de múltipla escolha, por meio das quais foram obtidos dados sociodemográficos e opiniões e percepções desses indivíduos quanto aos métodos de ensino-aprendizagem utilizados pelo corpo docente do curso de Medicina da UERN.

Para fins de análise dos dados obtidos, consideramos a aula expositiva, aulas práticas/de campo, discussão de casos clínicos e seminários como métodos tradicionais de ensino-aprendizagem para o curso de Medicina. Como métodos contemporâneos de ensino foram consideradas as atividades lúdicas, mapas conceituais, estudos dirigidos, aprendizagem baseada em problemas (ABP) e aprendizagem baseada em equipe $(\mathrm{ABE})$.

Os dados obtidos foram codificados, inseridos numa planilha eletrônica no software Microsoft Excel, de acesso exclusivo aos membros executores da pesquisa e mantidos em completo sigilo e anonimato.

As análises descritivas da população estudada foram feitas mediante apresentação de tabelas e gráficos de frequências. Os resultados, quando apropriado, foram submetidos a avaliação estatística por meio do teste do $\chi 2$ de Pearson ou Exato de Fisher, utilizando-se os softwares Pepi ${ }^{9}$ e Past $^{10}$. Foram considerados como significantes valores de $\mathrm{p} \leq 0,05$.

Os aspectos subjetivos da pesquisa, coletados nas entrevistas por meio das perguntas abertas, foram avaliados pela metodologia de "nuvem de palavras", uma ferramenta prática capaz de identificar os termos mais frequentes de um texto e destacá-los mediante a seleção automática do tamanho da fonte, em função da frequência, auxiliando, assim, na identificação de termos emergentes e contribuindo para desvendar as categorias empíricas. Para isso, cada item aberto do questio- 
nário foi analisado separadamente, gerando "nuvens de palavras" independentes. Todas as respostas de determinado item da entrevista foram agrupadas e importadas para o software WordArt ${ }^{11}$. Nesse processo, foram incluídos todos os termos que expressavam a subjetividade dos participantes e excluídos os termos considerados ruídos: artigos, pronomes, acentuações e os demais termos e/ou expressões que não manifestassem a subjetividade do respondente em relação ao objeto da pesquisa. Além disso, como as respostas aos questionários dependem diretamente da escolha de palavras individuais de cada entrevistado, citações diferentes com o mesmo significado foram padronizadas numa única terminologia, visando também a maior acurácia dessa análise subjetiva. $\mathrm{O}$ resultado final dessa análise permitiu construir duas nuvens de palavras diferentes, cada uma com suas particularidades de organização e diagramação, porém todas de fácil identificação e interpretação.

$\mathrm{O}$ presente estudo teve seus aspectos éticos avaliados e aprovados pelo Comitê de Ética em Pesquisa da Universidade do Estado do Rio Grande do Norte (CEP-UERN - Parecer $n^{\circ}$ 1.108.631 de 30/06/2015; CAAE - 43766115.0.0000.5294) e foi desenvolvido seguindo os princípios éticos de pesquisa com seres humanos, conforme descrito na Declaração de Helsinki.

\section{RESULTADOS}

A aula expositiva e outros métodos de ensino tradicionais foram as técnicas didático-pedagógicas mais utilizadas em sala de aula segundo os docentes e discentes entrevistados. Outras técnicas contemporâneas de ensino, como ABP, atividades lúdicas e $\mathrm{ABE}$, também foram relatadas, mas em percentual bem inferior (todos abaixo de 35,0\%) (Figura 1). Além de mais utilizadas, as metodologias tradicionais também foram consideradas as mais efetivas para o processo de ensino-aprendizagem pela maioria dos docentes $(62,78,0 \%)$ e discentes $(129,92,0 \%)$ entrevistados.

No entanto, grande parte dos discentes (103, 73,57\%) afirmou que gostaria que os professores utilizassem outros métodos didático-pedagógicos em suas aulas, tendo como justificativa mais citada a melhora do aprendizado em geral (Figura 2A). De modo semelhante, boa parte dos docentes $(60,75,0 \%)$ também afirmou que gostaria de usar outras metodologias de ensino, mas a falta de experiência e capacitação pedagógica constituiu o obstáculo mais comumente relatado $(28,29,4 \%)$ para a implementação de novas técnicas didáticas em suas aulas (Figura 3). Entre as principais sugestões dos docentes para a melhoria do processo ensino-aprendizagem no curso de Medicina, a mais citada foi a promoção de capacitação didático-pedagógica (Figura 2B).
Figura 1

Principais técnicas didático-pedagógicas utilizadas pelos professores do curso de Medicina da UERN em suas aulas/atividades curriculares entre julho de 2015 e junho de 2016.

ABP: Aprendizagem Baseada em Problemas; ABE: Aprendizagem Baseada em Equipe

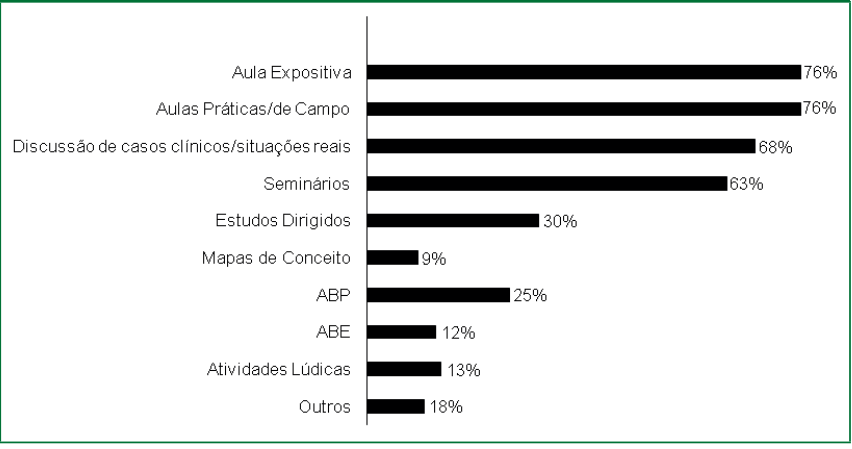

Observou-se que maior tempo de graduação e de experiência docente, formação em outros cursos de graduação da área da saúde e titulação em nível de pós-graduação stricto sensu se mostraram significativamente associados à utilização de métodos contemporâneos de ensino. A realização de outros níveis/cursos de capacitação pedagógica ficou no limite da significância estatística (Tabela 1).

\begin{tabular}{|c|c|c|c|}
\hline \multirow{3}{*}{ Perfil Docente } & \multicolumn{2}{|c|}{$\begin{array}{l}\text { Associação do perfil docente do curso } \\
\text { de Medicina da UERN com o uso de } \\
\text { hetodologias contemporâneas de ensino }\end{array}$} & \multirow{3}{*}{$\mathbf{P}$} \\
\hline & \multicolumn{2}{|c|}{$\begin{array}{l}\text { Uso de Metodologias } \\
\text { Contemporâneas }\end{array}$} & \\
\hline & Não & Sim & \\
\hline \multicolumn{3}{|l|}{ Anos de docência } & \multirow{4}{*}{$0,046^{*}$} \\
\hline Até 3 & $13(76,5 \%)$ & $6(35,3 \%)$ & \\
\hline $4-6$ & $9(60,0 \%)$ & $6(40,0 \%)$ & \\
\hline 7 ou mais & $16(33,3 \%)$ & $32(66,7 \%)$ & \\
\hline \multicolumn{3}{|l|}{ Graduação } & \multirow{3}{*}{$0,020^{*}$} \\
\hline Medicina & $37(56,9 \%)$ & $28(43,1 \%)$ & \\
\hline Outros cursos & $3(20,0 \%)$ & $12(80,0 \%)$ & \\
\hline \multicolumn{3}{|c|}{ Tempo de graduação } & \multirow{4}{*}{0,103} \\
\hline$\leq 5$ anos & $1(33,3 \%)$ & $2(66,7 \%)$ & \\
\hline $6-15$ anos & $19(65,5 \%)$ & $10(34,5 \%)$ & \\
\hline$\geq 16$ anos & $20(41,7 \%)$ & $28(58,3 \%)$ & \\
\hline \multicolumn{3}{|l|}{ Pós-graduação } & \multirow{3}{*}{$0,002^{*}$} \\
\hline Lato sensu & $33(63,5 \%)$ & $19(36,5 \%)$ & \\
\hline Stricto sensu & $7(25,0 \%)$ & $21(75,0 \%)$ & \\
\hline \multicolumn{3}{|c|}{ Capacitação pedagógica } & \multirow{3}{*}{0,070} \\
\hline Não & $21(61,8 \%)$ & $13(38,2 \%)$ & \\
\hline Sim & $19(41,3 \%)$ & $27(58,7 \%)$ & \\
\hline
\end{tabular}

P: P valor calculado pelo teste do Qui-Quadrado, ou Exato de Fisher (quando apropriado)

* Estatisticamente significativo. 


\section{Figura 2}

Nuvens de palavras com as justificativas dos discentes

do curso de Medicina da UERN para o desejo de

utilização de outros métodos de ensino-aprendizagem

pelos professores (A) e com as sugestões dos

docentes para $o$ aprimoramento do processo ensino-

aprendizagem do curso de Medicina da UERN (B)

A)

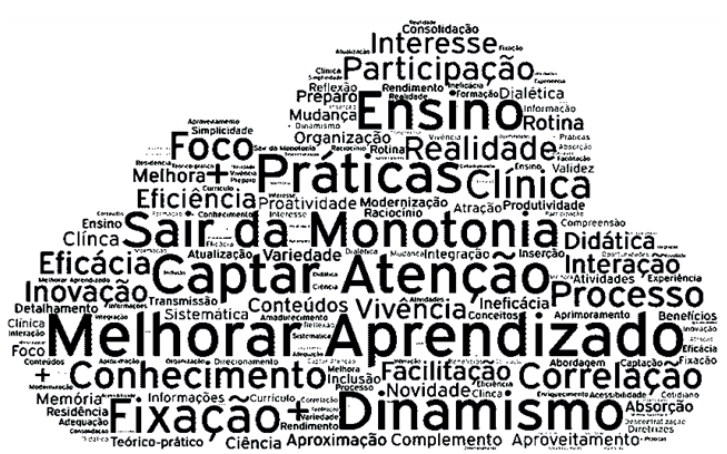

B)

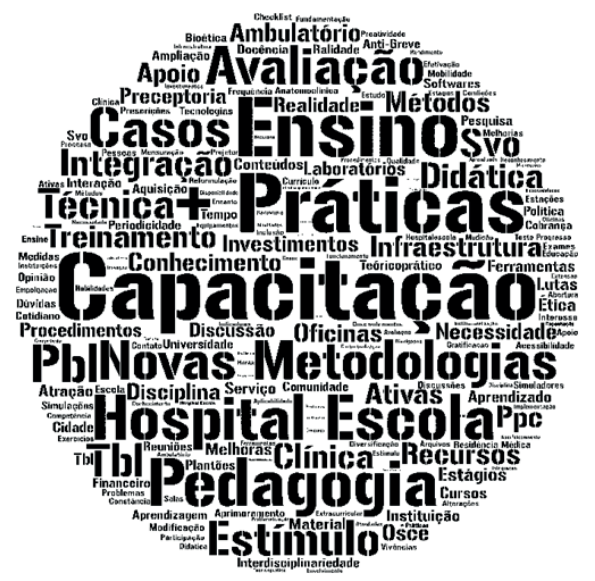

Figura 3

Principais obstáculos relatados pelos professores do curso de Medicina da UERN para a utilização de outras técnicas didático-pedagógicas

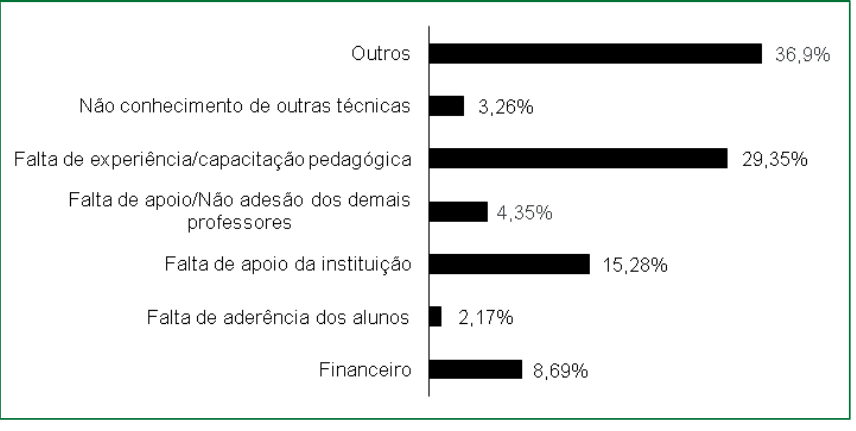

\section{DISCUSSÃO}

As qualidades demandadas na formação dos profissionais da área da saúde apontam um novo modo de entendê-la e desenvolvê-la, o que repercute nas universidades, produzindo alterações na formulação de propostas pedagógicas e na organização do trabalho pedagógico. Entre as características do mundo contemporâneo que provocam discussões sobre a formação para o trabalho em saúde, incluem-se: a velocidade da produção de conhecimento; uma necessária reflexão sobre a inserção do profissional de saúde nesse novo contexto; e a influência dos meios de comunicação na construção do profissional, que está afastado da reflexão sobre a vida, da inserção no mundo e da configuração de nova organização do espaço social, o que exige postura crítica ${ }^{7}$.

Assim como esse novo contexto do mundo contemporâneo exige mudanças no processo de ensino-aprendizagem, a divulgação das novas DCN do curso de graduação em Medicina também o faz. De acordo com as DCN, os cursos de Medicina devem utilizar metodologias ativas como uma das ferramentas de ensino, as quais auxiliam na formação geral, humanista, crítica, reflexiva e ética do graduando ${ }^{12}$. O projeto pedagógico do curso (PPC) de Medicina da UERN contempla esta pedagogia ativa e centrada no aluno como orientação metodológica e tem como foco o processo saúde-doença. O uso de procedimentos, métodos e técnicas de ensino variados é vislumbrada no PPC com o objetivo de capacitar o corpo discente para a análise do problema real e para a tomada de decisões exequíveis ${ }^{13}$.

No presente estudo se percebeu, no entanto, o predomínio do modelo tradicional de ensino no curso de Medicina da UERN. Introduzido há mais de cem anos pelo relatório Flexner, esse modelo tradicional tinha como mérito a busca da excelência na preparação dos futuros médicos, introduzindo uma salutar racionalidade científica para o contexto do século XX. Todavia, desconsiderava outros fatores que afetam profundamente os impactos da educação médica na prática profissional e na organização dos serviços de saúde ${ }^{14}$. $\mathrm{O}$ docente desempenharia o papel de transmissor de conteúdos por meio de aulas expositivas para grandes grupos sob a forma de palestras ou conferências, com uma ampla quantidade de informações e ênfase na valorização do conteúdo transmitido. Já os estudantes se limitam a absorver e repetir os conteúdos transmitidos, por meio de uma atitude espectadora e passiva, com pouca autonomia em seu aprendizado ${ }^{15}$.

A predominância dos métodos tradicionais de ensino-aprendizagem observados no curso de Medicina da UERN reflete, portanto, um descompasso entre a práxis educacional e os referenciais atuais que norteiam a formação do profissio- 
nal médico. O uso de metodologias integradoras e a criação de outras oportunidades de aprendizagem possibilitam ampliar as experiências para além do ambiente hospitalar e das instituições formais. Deste modo, sua utilização potencializa o desenvolvimento da capacidade comunicativa e de articulações, partindo das questões reais de saúde das comunidades, além de permitir e estimular a atuação interprofissional e a construção coletiva de alternativas de melhorias ${ }^{12,16}$.

Em comparação com os métodos contemporâneos, os métodos tradicionais de ensino também foram considerados mais efetivos para o processo de ensino-aprendizagem por grande parte dos entrevistados. Estes resultados foram semelhantes aos obtidos com estudantes do curso de Medicina da Universidade Federal de Goiás, Goiânia (GO). Em seu trabalho, Deus et al. ${ }^{17}$ relatam que a metodologia tradicional foi a que obteve maior aceitabilidade e a que melhor correspondeu às expectativas dos estudantes, embora a abordagem mais ativa e participativa analisada tivesse sido associada a melhores resultados em termos de frequência, atenção e interatividade. Em outro estudo, Silva et al ${ }^{18}$ relatam que com a metodologia ativa há uma valorização da troca de experiências em sala de aula, atuação e acompanhamento dos professores e esclarecimento das dúvidas em conjunto. Em contrapartida, apesar de ter boa receptividade, ela não é bem-vista por todos os discentes, principalmente por encontrarem parâmetros diferenciados de avaliação e de participação em sala de aula. Entre as limitações apontadas por discentes como deficiências da metodologia, destacam-se a falta de avaliações escritas e a falta de confiança na aprendizagem somente por meio do diálogo problematizador $^{18}$.

No entanto, um trabalho mais recente realizado por Fermozelli et al. ${ }^{19}$ mostrou que a maioria dos discentes relatou aumento de interesse pelos estudos com a utilização de uma metodologia ativa - ensino híbrido, blended learning, que utiliza estudos à distância seguidos de aulas práticas face a face - na disciplina de Patologia, e que esta abordagem promoveu maior integração entre os conteúdos trabalhados e a clínica dos pacientes ${ }^{19}$. Resultados de metanálise realizada recentemente também mostraram que os métodos e atividades de aprendizado em pequenos grupos eram, em média, mais eficazes do que a instrução tradicional baseada em conferências, com efeito médio ponderado significativo ${ }^{20}$.

A preferência pelas técnicas didático-pedagógicas mais tradicionais entre os docentes e discentes do curso de Medicina provavelmente reflete a pouca vivência dos professores com as metodologias ativas de aprendizagem e, consequentemente, a dificuldade em colocá-las em prática e de forma adequada durante as aulas. Corrobora esta hipótese o desejo de $75,0 \%$ do corpo docente de utilizar outros métodos - o que não ocorre principalmente pela falta de experiência com estas técnicas -, além das associações significativas observadas entre a utilização de metodologias mais contemporâneas e a formação em nível de pós-graduação stricto sensu e anos de docência universitária. Além disso, a grande maioria dos discentes também manifestou a vontade de que os professores utilizassem outros métodos didático-pedagógicos em suas aulas, e o argumento mais utilizado para isso era a melhora do aprendizado.

Além das competências específicas para exercer a profissão, a docência universitária exige a aquisição/aprendizado de habilidades relacionadas especificamente ao ensino superior, como o domínio de uma área do conhecimento, o domínio pedagógico e o exercício da dimensão política, assim como habilidades cognitivas e não cognitivas, entre elas boa comunicação com os alunos, didática, empenho, envolvimento em pesquisas, atualização constante, organização, autocrítica, capacidade de fornecer feedback aos discentes e de integrar teoria e prática ${ }^{21,22}$.

Freitas et al. ${ }^{23}$ explanam que o desenvolvimento docente representa um desafio que persiste não apenas em escolas médicas, como também em outros cursos da área de saúde, representando uma demanda, inclusive entre mestres e doutores com longa experiência de ensino. E mesmo na presença de indícios de quebra da hegemonia do modelo tradicional, existem limitações que refletem características do próprio processo e programas formativos. Por mais que os professores busquem incorporar novos saberes e abordagens inovadoras em suas práticas, estas são muito mais direcionados à formação científica do que à formação pedagógica. Além disso, a área da saúde tradicionalmente atribui maior valor à produção científica do que à formação pedagógica ${ }^{23}$.

Percebe-se, assim, a necessidade e importância da educação pedagógica continuada por meio da manutenção permanente do Programa de Formação e Desenvolvimento da Docência em Saúde preconizado nas novas Diretrizes Curriculares Nacionais do curso de graduação em Medicina, com vistas à valorização do trabalho docente na graduação e ao maior envolvimento dos professores com o projeto pedagógico do curso, por meio de domínio conceitual e pedagógico que englobe estratégias de ensino mais contemporâneas ${ }^{12}$.

Recentemente, duas iniciativas propostas por docentes do corpo efetivo foram adotadas na tentativa de minimizar as carências apontadas acima na capacitação docente: a primeira foi o I Seminário Regional de Educação Médica (Semed), em 2016, que discutiu a reforma curricular e possibilitou captar as demandas de qualificação; e a segunda, o I Curso de Aper- 
feiçoamento Docente em Processos Pedagógicos em Saúde para uma Formação Baseada em Competências, em 2017. Iniciativas como essas denotam que os docentes compreendem a importância da qualificação no processo de implementação das diretrizes e que buscam suprir essa necessidade, inclusive com iniciativas próprias. No entanto, para maior impacto, essas ações devem atingir um patamar de continuidade e sustentabilidade, o que poderá ser favorecido pelo apoio institucional e pela valorização, existindo diversas opções para abordar estes aspectos e que devem ser discutidas.

A mudança do método de ensino tradicional para o ativo normalmente gera insegurança, requer grande esforço dos atores envolvidos no processo e exige maturidade, organização dos estudantes e mudança de comportamento para assumir a responsabilidade sobre o próprio aprendizado. Os alunos ainda podem se sentir perdidos, tendo em vista que eles mesmos precisam buscar conhecimento das áreas básicas antes de aprofundar as questões clínicas dos pacientes, o que nem sempre é seguido na prática e pode ser ignorado por pressa, falta de organização ou mesmo por não se saber até aonde ir e quando parar frente à complexidade de certos temas ${ }^{24}$.

A carga horária elevada do curso de Medicina na UERN - 7.935 horas em disciplinas obrigatórias, 225 horas em disciplinas optativas, 1.530 horas em atividades complementares - pode ser outro fator que tenha dificultado a utilização e adesão dos discentes às atividades exigidas pelas metodologias contemporâneas, provocando certa aversão pelo desenvolvimento destas atividades, que representariam maior investimento de tempo.

\section{CONCLUSÕES}

A metodologia tradicional foi considerada a mais efetiva e predominante no curso de Medicina da UERN, embora outras experiências pedagógicas tenham sido relatadas. A falta de experiência e habilidade didático-pedagógica docente pode ter contribuído sobremaneira para dificultar a implementação e aceitação das metodologias contemporâneas pelos professores. Além disso, a carga horária elevada do curso e a falta de vivência nestas metodologias podem levar à aversão dos alunos a esses métodos.

Entretanto, é necessário impulsionar o desenvolvimento de metodologias centradas no aluno por meio da adoção de pedagogias ativas de ensino e estimular a capacitação didático-pedagógica do corpo docente com vistas a promover a implementação do projeto pedagógico do curso, em especial nos cursos da área da saúde, como o de Medicina.

\section{REFERÊNCIAS}

1. Mello CCB, Alves RO, Lemos SMA. Metodologias de ensino e formação na área da saúde: revisão de literatura. Rev. CEFAC. 2014; 16(6):2015-2028.

2. Gomes AP, Rego S. Transformação da Educação Médica: É Possível Formar um Novo Médico a partir de Mudanças no Método de Ensino-Aprendizagem? Rev Bras Educ Med. 2011; 35(4):557-566

3. Mendonça ET, Cotta RMM, Lelis VP, Carvalho Junior PM. Paradigms and trends in higher education: the action research methodology as a teacher education strategy. Interface (Botucatu). 2015; 19(53)373-86.

4. Cezar PHN, Guimarães FT, Gomes AP, Rôcas G, Siqueira-Batista R. Transição paradigmática na educação médica um olhar construtivista dirigido à aprendizagem baseada em Problemas. Rev Bras Educ Med. 2010;34(2):298-303.

5. Heimann C. Capacitação pedagógica de docentes de enfermagem: desenvolvimento e avaliação de um curso à distância. São Paulo; 2012. Mestrado [Dissertação] - Universidade de São Paulo.

6. McMahon GT. What Do I Need to Learn Today? - The Evolution of CME. N Engl J Med. 2016;374(15):1403-1406.

7. Villardi ML, Cyrino EG, Berbel NAN. Mudança de paradigma no ensino superior em saúde e as metodologias problematizadoras. In: A problematização em educação em saúde: percepções dos professores tutores e alunos [online]. São Paulo: Editora UNESP; 2015. p. 23-44.

8. Souza CS, Iglesias AG, Pazin-Filho A. Estratégias inovadoras para métodos de ensino tradicionais - aspectos gerais. Medicina (Ribeirão Preto). 2014. 47(3): 284-92.

9. Gahlinger PM, Abramson JH. Computer programs for epidemiologic analysis: PEPI. Stone Mountain, GA, USD Inc, 1995.

10. Hammer $\varnothing$, Harper DAT, Ryan PD. PAST: Paleontological Statistics Software Package for Education and Data Analysis. Palaeontologia Electronica. 2001.4(1): 9pp.

11. WORD ART. Tagul: WordArt.com. 2016. Disponível em: https: / /tagul.com. Acesso em: 14 maio 2016.

12. Brasil. Ministério da Educação. Conselho Nacional de Educação. Câmara de Educação Superior. Resolução CNE/ CES 3/2014 nº 3 de 20 de junho de 2014. Institui Diretrizes Curriculares Nacionais do Curso de Graduação em Medicina e outras providências. Diário Oficial da União. Brasília, 23 jun. 2014; Seção 1, p. 8-11.

13. UERN. Projeto Pedagógico do Curso de Medicina da Faculdade de Ciências da Saúde. Disponível em: http:/ / www. uern.br / controledepaginas / documentos-legislacao-ensino/arquivos/0065ad_referendum_consepe_6_2014_ 
aprova_o_ppc_de_medicina_do_campus_central_anexos. pdf. Acesso em: 09 de março 2018.

14. Pagliosa FL, Da Ros MA. O Relatório Flexner: Para o Bem e Para o Mal. Rev Bras Educ Med. 2008.32 (4) : 492-499.

15. Prevedello AS, Segato GF, Emerick LBBR. Metodologias de ensino nas escolas de medicina e a formação médica atual. Rev. Educ., Cult. Soc.2017.7(2):566-577.

16. WHO. Transforming and scaling up health professionals' education and training. World Health Organization Guidelines 2013; [capturado 31 maio 2018]. Disponível em: http://apps.who.int/iris/bitstream/handle/10665/93635/9789241506502_eng.pdf;jsessionid=8DC 479ED8DE34319922740E01FD65E4B? sequence =1

17. José Miguel de Deus JM, Nonato DR, Alves RBF, Silva MMM, Amaral AF, Bollera VR. Aula Centrada no aluno versus aula Centrada no Professor. Desafios para mudança. Rev Bras Educ Med. 2014. 38 (4):419-426.

18. Silva JLL, Assis DL, Gentile AC. A percepção de estudantes sobre a metodologia problematizadora: a mudança de paradigma em relação ao processo ensino-aprendizagem. Rev. eletrônica enferm. [on line]. 2005. 07(01) [capturado 05 fev. 2018]; 72-80. Disponível em: https://revistas.ufg.br/ fen/article/view/852

19. Fermozelli JÁ, Cesaretti MLR, Barbo MLP. Blended learning strategies in teaching general pathology at a medical course. J. Bras. Patol. Med. Lab. 2017. 53(03):202-209.

20. Kalaian SA, Kasim RM. Effectiveness of various innovative learning methods in health science classrooms: a meta-analysis. Adv Health Sci Educ Theory Pract. 2017;22(5):1151-1167.

21. Costa NMSC. Formação pedagógica de professores de medicina. Rev. Latino-Am. Enfermagem [on line]. 2010; 18(1) [capturado 03 fev. 2018]; 07 telas. Disponível em: http:/ / www.scielo.br/pdf/rlae/v18n1/pt_16.pdf

22. Belfor JA, Sena IS, da Silva DKB, Lopes BRS, Júnior MK, dos Santos BEF. Competências pedagógicas docentes sob a percepção de alunos de medicina de universidade da Amazônia brasileira. Ciência \& Saúde Coletiva. 2018. 23(1):73-82.
23. Freitas DA, Santos EMS, Lima LVS, Miranda LN, Vasconcelos EL, Nagliate PC. Saberes docentes sobre processo ensino-aprendizagem e sua importância para a formação profissional em saúde. Interface (Botucatu). 2016; 20(57):437-48.

24. Marin MJS, Lima EFG, Paviotti AB, Matsuyama DT, Silva LKD, Gonzalez C, et al. Aspectos das fortalezas e fragilidades no uso das Metodologias Ativas de Aprendizagem. Rev Bras Educ Med. 2010. 34(1):13-20.

\section{CONTRIBUIÇÃO DOS AUTORES}

Poliana Oliveira da Cruz: concepção e desenho do estudo; coleta, tabulação e análise de dados; escrita do manuscrito. Thaís Bandeira de Carvalho: coleta, tabulação e análise de dados; escrita do manuscrito. Luca Di Pace Pinheiro: coleta, tabulação e análise de dados. Patrícia Estela Giovannini: análise dos dados; escrita e revisão crítica do manuscrito. Ellany Gurgel Cosme do Nascimento: análise dos dados; escrita e revisão crítica do manuscrito. Thales Allyrio Araújo de Medeiros Fernandes: concepção e desenho do estudo; análise dos dados; escrita e revisão crítica do manuscrito.

\section{CONFLITO DE INTERESSES}

Os autores declaram que não possuem qualquer conflito de interesses.

\section{ENDEREÇO PARA CORRESPONDÊNCIA}

Poliana Oliveira da Cruz

Universidade do Estado do Rio Grande do Norte

Departamento de Ciências Biomédicas

Rua Miguel Antônio da Silva Neto, s/n ${ }^{\circ}$

Aeroporto - Mossoró

CEP 59607-360 - RN

E-mail: poliana.oliveira.cruz@gmail.com 